\title{
Methods to Trap High-Temperature Conformations of Proteins using Fast Cooling M McLeod ${ }^{1}$, R Thorne ${ }^{2}$, T Holyoak ${ }^{3}$ \\ ${ }^{1}$ Cornell University ${ }^{2}$ Physics Dept, Cornell Univ, ${ }^{3}$ Biology Dept, Univ of Waterloo \\ mjmcleod@uwaterloo.ca
}

Enzyme function shows a trend of increasing activity with temperature. On an Arrhenius plot, this relationship shows a near linear variation up to a maximum temperature, beyond which activity drops and the enzyme has been assumed to thermally denature. Recently, more complex temperature variation has been observed, in which Arrhenius plots show increasing downward curvature with increasing temperature. Furthermore, after the activity maximum is reached, the enzyme may come reversibly inactive although not thermally denatured. These hightemperature states are largely undescribed, and the origins of the observed kinetic changes are still contentious. Structural insight into these states may yield valuable information describing a proteins change in free-energy landscape with temperature and could further describe the intricate evolutionary changes that occur as an organism adapts to varying thermal niches. Although room-temperature crystallography is becoming easier with the advent of fast-readout detectors and vector scanning at synchrotron sources, these experiments are still very challenging. The difficulty in observing these high-temperature structural states harken back to impetus in the development of cryocrystallography. First, radiation damage becomes a significant problem at temperatures above the glass transition where free radical are able to migrate. Second, crystals may not be amenable to be held at temperatures above those at which they were crystallized. In addition, other quality-of-life features of cryocrystallography are not accessible such as samples storage and use of mail-in synchrotron programs. The methods currently being developed in the Thorne lab are aiming to marry the benefits of cryocrystallography, but also alleviate potential artifacts of the method shown to change the structure of some proteins induced by slow cooling using typical practices. In the presented work, we will showcase our current solution to trapping high-temperature states by equilibrating crystals at a target temperature followed by rapid cooling, to evaluate the structural states of a metabolic enzyme phosphoenolpyruvate carboxykinase that has been extensively studied in the Holyoak lab. This enzyme has been observed to have non-linear Arrhenius temperature dependencies and has both a high-diffracting mesophilic and psychrophilic enzymes. This new structural information in conjunction with the vast biochemical and kinetic data will hopefully begin to illuminate the origins of the temperature dependencies of proteins. 